\title{
Contrast enhanced ultrasonography in assessing the treatment response to transarterial chemoembolization in patients with hepatocellular carcinoma.
}

\author{
Zeno Spârchez ${ }^{1,2}$, Tudor Mocan ${ }^{1}$, Pompilia Raduํ, Ofelia Anton${ }^{1}$, Mihai Comsa ${ }^{1}$, Tudor Vasile ${ }^{1}$, \\ Nicolae Bolog 3
}

${ }^{1}$ Institute for Gastroenterology and Hepatology, Cluj-Napoca, Romania, ${ }^{2} 3^{\text {rd }}$ Medical Department, "Iuliu Haţieganu" University of Medicine and Pharmacy, Cluj-Napoca, Romania, ${ }^{3}$ Phoenixswissmed, Basel, Switzerland

\begin{abstract}
The last decades have known continuous development of therapeutic strategies in hepatocellular carcinoma (HCC). Unfortunately the disease it still not diagnosed until it is already at an intermediate or even an advanced disease. In these circumstances transarterial chemoembolization (TACE) is considered an effective treatment for HCC. The most important independent prognostic factor of both disease free survival and overall survival is the presence of complete necrosis. Therefore, treatment outcomes are dictated by the proper use of radiological imaging. Current guidelines recommend contrast enhanced computer tomography (CECT) as the standard imaging technique for evaluating the therapeutic response in patients with HCC after TACE. One of the most important disadvantage of CECT is the overestimation of tumor response. As an attempt to overcome this limitation contrast enhanced ultrasound (CEUS) has gained particular attention as an imaging modality in HCC patients after TACE. Of all available imaging modalities, CEUS performs better in the early and very early assessment of TACE especially after lipiodol TACE. As any other imaging techniques CEUS has disadvantages especially in hypovascular tumors or in cases of tumor multiplicity. Not far from now the current limitations of CEUS will be overcome by the new CEUS techniques that are already tested in clinical practice such as dynamic CEUS with quantification, three-dimensional CEUS or fusion techniques.

Keywords: hepatocellular carcinoma, contrast enhanced ultrasound, transarterial chemoembolization, contrast enhanced computer tomography, magnetic resonance imaging
\end{abstract}

\section{Introduction}

Hepatocellular carcinoma (HCC) is still the most common primary neoplasia of the liver. There have been tremendous efforts in the development of therapeutic strategies. Unfortunately during the last 30 years, patient survival has not been improved as greatly as for many other cancers, basically because the disease it still not diagnosed until it is already at an intermediate or even an advanced disease [1]. Moreover, it should also take into consideration that the majority of HCCs appears on the background

Received 05.01.2016 Accepted 20.01.2016

Med Ultrason

2016, Vol. 18, No 1, 96-102

Corresponding author: Dr. Tudor Mocan

Institute for Gastroenterology and Hepatology

Croitorilor 19-21,

Cluj-Napoca, Romania

Email: mocan_tudor@yahoo.com of a cirrhotic liver. Treatment algorithms depend on both the stage of HCC and the underling liver function [2].

In these circumstances, the last decade has brought significant advancement in the management of the disease. Many new treatment techniques are now available. Of these, transarterial chemoembolization (TACE) has gained more and more attention, particularly on a subset of HCC patients not suitable for liver transplantation, resection or ablation. Nowadays TACE is considered an effective treatment for HCC [3].

Complete necrosis after TACE is definitely an independent prognostic factor of both disease free survival and overall survival. Since histopathologic examination of each nodule is neither feasible nor reasonable for HCC patients, treatment outcomes are dictated by the use of radiological imaging [4]. Hence, after TACE has been performed, evaluation with imaging is crucial to determine whether there is or not complete necrosis. In cases of partial necrosis a new TACE 
session or additional treatment such as ethanol injection, radiofrequency or microwave ablation may be performed in an attempt to achieve complete tumor necrosis [5].

Contrast enhanced computer tomography (CECT) is commonly used as the standard imaging technique for evaluating the therapeutic response in patients with $\mathrm{HCC}$ after TACE. The technique has some disadvantages, one of the most important being the overestimation of tumor response [6]. In order to overcome these limitations, many researchers have focused on other imaging techniques. Within these, contrast enhanced ultrasound (CEUS) with second-generation contrast agents (SonoVue) has gained particular attention as an imaging modality in HCC patients after TACE.

According to the American Association for the Study of Liver Diseases (AASLD), CEUS is not recommended anymore in the diagnosis algorithm because it may offer false positive HCC diagnosis in patients with cholangiocarcinoma [7]. Despite these recommendations in Europe CEUS is extensively used in the diagnosis of HCC basically because of the higher experience. Some authors consider the combination of CEUS and multidetector CT (or dynamic MR imaging) as the most rational and cost-effective combination for the noninvasive diagnosis of small HCC [8].

CECT is still considered the gold standard when evaluating the response of ablation techniques in HCC. Even in this scenario, many authors have suggested that CEUS should be the first imaging technique to evaluate the initial response (1 month) and CT and MRI should be reserved for follow-up at 3 months [9]. Moreover an early assessment (immediately after the procedure or the next day) is very important since it may allow detection of areas not covered by the treatment, which will need a second treatment through a new insertion. With the use of CEUS the second insertion could be performed during the same session. Hence, the same operator using the same equipment, thus avoiding more expensive methods, could do both the ablation and evaluation of efficacy. Using this approach, it is possible to decrease the rate of partial necrosis in treated HCCs from $16.1 \%$ to $3.8 \%$ [10]. A decrease of partial necrosis stands for increase disease free and overall survival.

The purpose of this review was to present the ability of CEUS to evaluate treatment results after TACE in HCC patients. Possible applications of CEUS during and after TACE and new CEUS techniques will be discussed. The major advantages of CEUS over other imaging techniques will be provided based on our experience and currently available literature.

\section{CEUS as a tool to evaluate the efficacy of TACE}

a) imaging findings. Positive enhancement is defined as strong contrast enhancement appearing within the tu- mor. Positive enhancement is interpreted as viable tumor in treated nodules and considered an incomplete response to TACE (fig 1). In rare situations, the whole nodule is enhancing, in those cases TACE is considered without response (fig 2). No enhancement of the lesion is defined as no-bubble signal within the tumor while the surrounding liver parenchyma was filled with bubbles signals. No enhancement is considered complete tumor necrosis or complete response [11-13] (fig 3). Sometimes the necrot-

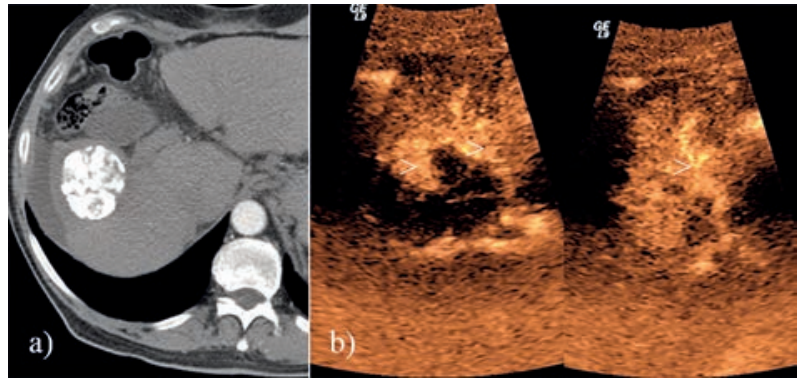

Fig 1. a) Incomplete response after TACE. Arterial phase of a follow up CT after the first session of TACE depicts an important deposition of lipiodol in a large HCC with some peripheral areas without retention of iodized oil; b) CEUS in 2 different planes shows a peripheral enhancement within the lesion $(>)$.

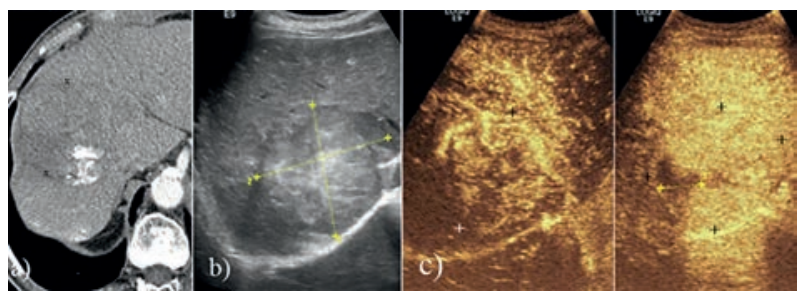

Fig 2. a) No response after TACE. Arterial phase of a CT performed after 2 months shows scars deposits of lipiodol $(>)$ in a large, enhancing HCC; b) US shows a $6 \mathrm{~cm}$ inhomogeneous tumors in the $8^{\text {th }}$ segment; c) CEUS shows an almost complete enhancement of the tumor.

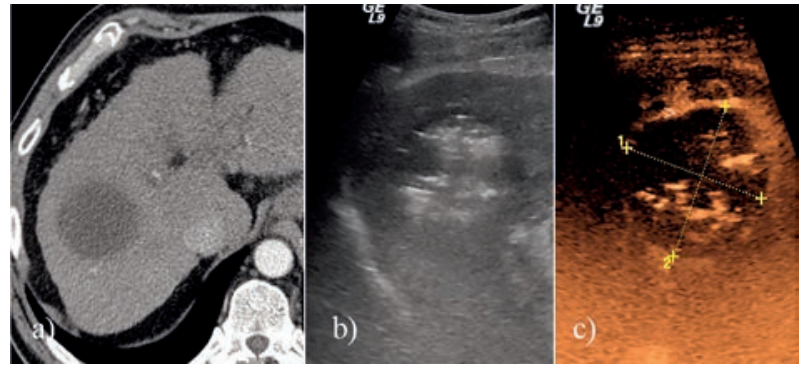

Fig 3. Complete response after a DEB-TACE. a) CT in arterial phase performed one month after TACE demonstrates the complete absence of enhancement; b) US performed 1 week after TACE revealed the presence of echogenic material inside de lesions. c) CEUS evaluation was disturbed by the echogenic material but showed no enhancement within the lesion. Note a rim like enhancement $(>)$ around the treated HCC. 


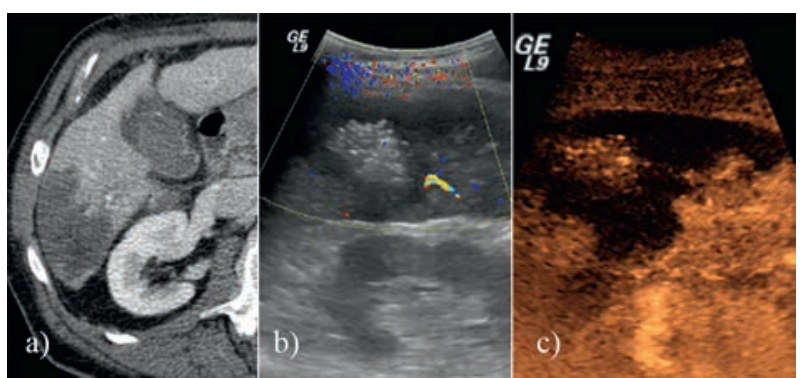

Fig 4. Hepatic infarction around an HCC after a DEB-TACE: a) a larger area of necrosis in respect to the tumor is seen on CT scan in portal phase; b) US showed echogenic material inside the tumors and a hypoechoic triangular area towards the liver capsula. No vessels were depicted in that area by color Doppler US. c) CEUS revealed a non-enhancing area around the tumor extending to the capsula.

ic area is larger than the tumor, in that case a partial/total infarct of a liver segment may occur (fig 4). We consider that the use of SonoVue might increase the specificity of CEUS for discerning viable and non-viable HCC after TACE.

b) timing of CEUS evaluation ( early vs. late evaluation). Currently, there is no consensus regarding which is the best interval for CEUS to be performed after TACE. It is well known that early HCC diagnosis offers better outcomes. Therefore, it is easy to assume that earlier diagnosis of incomplete necrosis after TACE could increase the overall survival. Kono et al were able to show that residual tumor blood flow on CEUS performed two or more days after TACE may predict tumor outcome that currently requires three months to be reliably detected on CT or MRI [14]. Other authors have shown that CEUS results 1 week after TACE were consistent with those of dynamic CT 2 months after intervention [15].

c) Intraprocedural CEUS during TACE. There are few studies assessing a possible role for intraprocedural CEUS. In one recent study intraprocedural CEUS underestimated the degree of necrosis in $50 \%$ of tumors completely devascularized on postembolization angiography [16]. This underestimation may be explained by the use of TACE with drug-eluting beads where tumor necrosis takes place during days or weeks after treatment. It should be also mentioned that intraprocedural CEUS might harbor a higher sensitivity in depicting tumor angiogenesis in comparison with angiography.

Intraarterial application of an sonographic contrast agent (SonoVue) using the placed intraarterial catheter is able to diagnose and locate additional hepatic lesions in patients with HCC, not detectable in the other applied imaging modalities [17]. These findings had a direct impact on patient management in almost two-thirds of all cases [17]. Intraprocedural CEUS with intra-arterial ul- trasound contrast agent injection may also help in finding extrahepatic tumor-feeding arteries [18]. However, the manufacturer does not indicate this route of administration, and further safety data are required before clinical translation. Although the number of patients was limited in both studies, there is some evidence to support the use of intraprocedural CEUS.

\section{Results of CEUS and comparison with TACE and MRI (including the results in various types of TACE: lipiodol, DEB)}

The sensibility and specificity of CEUS 1 week after TACE depends on the type of technique that was used as gold standard. Using histology as gold standard Morimoto $\mathrm{M}$ et al showed that CEUS has high sensitivity and specificity for discerning viable and non-viable HCC of $100 \%$ and $81 \%$ respectively [19]. A major limitation of that study is the use of Levovist, which is a first generation contrast agent with less harmonic features and stability in US field. Another study showed a sensitivity and specificity of $100 \%$ when findings of dynamic CT at 2 months after chemoembolization were regarded as the gold standard [5].

CECT is an image acquisition modality recommended by Modified Response Evaluation Criteria in Solid Tumors (mRECIST). Despite current recommendations, many investigators have shown a weakness of CECT in detecting residual tumors after lipiodol TACE.

A retrospective study including $130 \mathrm{HCC}$ patients compared CEUS with CECT performed 0.5-2 months after TACE in evaluating the treatment response using histology as gold standard [20]. The sensitivity, specificity and accuracy of CEUS vs. CECT were $95.9 \%(117 / 122)$ vs $76 \%(93 / 122) \quad(\mathrm{p}<0.001), 100 \% \quad(8 / 8)$ vs $100 \%$ $(8 / 8)(\mathrm{p}=1.000)$, and $96.2 \%(125 / 130)$ versus $77.7 \%$ $(101 / 130)(p<0.001)$, respectively [20]. Similar to these findings, Salvagio $\mathrm{G}$ et al using digital angiography as a gold standard showed that MDCT performed 1 month after treatment had a sensitivity of $86.9 \%$, specificity of $100 \%$, positive predicted value of $100 \%$, and negative predictive value of $83.3 \%$ in detecting residual tumors. In comparison, CEUS performed 1 month after treatment detected all the cases of incomplete response [11].

A more recent study evaluated the performance of CEUS, MDCT, and MRI in detecting residual tumors at 4 and 12 weeks after TACE. Taking the results of MRI at 12 weeks as reference, CEUS at 4 weeks has higher sensitivity than CECT (100\% vs. $50 \%)$ and even CEMRI (100\% vs.50\%) [21].

It is hazardous to draw same conclusions regarding these results but they definitely raise the question wheth- 


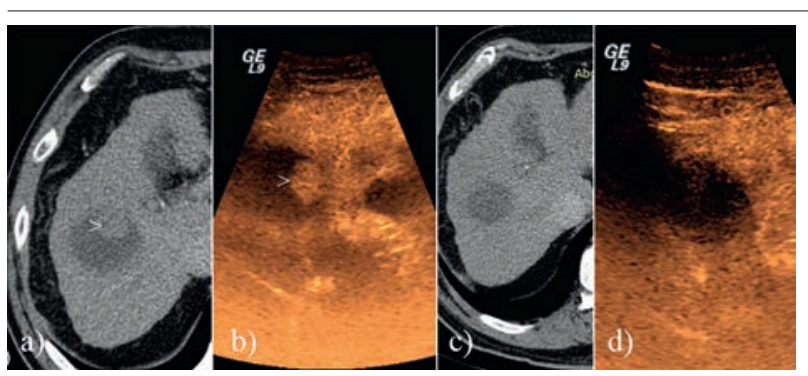

Fig 5. Intranodal recidive after TACE. CT (a) and CEUS (b) in arterial phase show an enhanced $2 \mathrm{~cm}$ lesion $(>)$ within the necrotic area; The pacient was treated by RFA. No residual tumor was found both on CT (c) and CEUS (d)

er CEUS is more sensitive than CT/MRI in detecting earlier the small areas of HCC.

Based on above-mentioned studies CEUS performed 1-4 weeks after lipiodol TACE is at least similar to CT performed 2 months after TACE [15]. The diagnosis of incomplete necrosis one week after TACE would allow additional treatment. CEUS guided radiofrequency or microwave ablation one week after TACE might transform the partial necrosis into complete necrosis and decrease the rate of recurrence (fig 5). There are already studies showing that TACE combined with radiofrequency ablation offers better outcomes compared to TACE alone [22].

New TACE concepts are based on drug-eluting beads and might be less susceptible to artifacts compared to Lipiodol, which can generate considerable artifacts in $\mathrm{CT}$ and may therefore change the diagnostic results [11]. Not surprisingly, CT was found comparable to MRI in a lipiodol free treatment group, meaning that both modalities produced comparable results in the absence of lipiodol [1]. It would be interesting to see if CEUS is still more accurate compared to CT in these new TACE concepts. Unfortunately, there are no studies comparing CECT with CEUS after DEB based TACE. Considering the half-life of doxorubicin in drug-eluting beads, a very early assessment may not be appropriate in the case of TACE with DC beads because the degree of necrosis could be underestimated [23]. However, all CEUS performed 1 to 2 weeks after DEB based TACE (a total of 14 tumors) resulted in $100 \%$ sensitivity, specificity, positive predicted value, negative predictive value, and accuracy when compared to contrast enhanced MRI/CT at 1 month [24]. Hence, even in these new TACE concepts, CEUS may identify patients requiring retreatment or additional treatment (radiofrequency or microwave ablation) earlier than the typical 4 weeks imaging.

It is worth to be mentioned that the use of lowmolecular-weight and water-soluble contrast agents in MRI makes it difficult to differentiate granulation tissue and residual tumor on one hand and peripheral viable tumors from inflammatory peritumoral infiltration on the other hand, when scanned within 4 weeks of treatment [25].

\section{mRECIST evaluation using CEUS and impact on survival}

mRECIST was introduced in 2008 to evaluate the response of HCC after loco regional (TACE, ablation) or anti-angiogenetic treatments [26]. It is based on unidimensional measurement of the enhancing parts of target tumors. New and growing evidence suggests that mRECIST is able to predict the overall survival in patients with HCC treated by TACE or Sorafenib [26].

CECT and CEMRI, are the standard modalities for an imaging assessment of tumor response in mRECIST [26]. In the last years CEUS was also used as imaging method in mRECIST evaluation after TACE in HCC patients [26]. In one recent study a high degree of concordance between CEUS and CT/MRI (kappa coefficient $=0.84$, $\mathrm{p}<0.001$ ) was demonstrated [26]. CEUS may be more reliable than CECT when evaluating a complete response by mRECIST after TACE [20]. Responders (complete + partial) had a significantly longer mean overall survival and time to progression compared to nonresponders (37.1 vs. 11.0 months, $\mathrm{p}<0.001$ and 24.6 vs. 10.9 months, $\mathrm{p}=0.007$, respectively) thus validating mRECIST-CEUS as a reliable prognostic tool [26].

\section{Advantages and limitations over TACE}

Some of the benefits of CEUS over CECT have already been discussed, the higher accuracy in detecting residual tumors at 1 week after TACE and lack of radiation being the most important.

CEUS is less expensive than CECT or CEMRI and can be performed repeatedly at bedside. The majority of HCC develop on the background of cirrhotic liver. Once that cirrhosis has developed, complications such as acute kidney injury are frequently encountered. In these circumstances the use of CEUS avoids the use of iodinated contrast agents. Moreover, in situations when repeated imaging is required after TACE, CT seems inappropriate due to the high risk of radiation $[12,13]$.

CEUS with SonoVue is able to detect smaller areas of viable HCC, which may be overlooked by $\mathrm{CT}$ or even MRI (fig 6), and also the patency of smaller vessels in early stage after TACE. Despite this ability, the clinical impact of this minimal residual tumor, in terms of survival, tumor recurrence and indication for prompt retreatment have not been investigated so far [11-13]. 


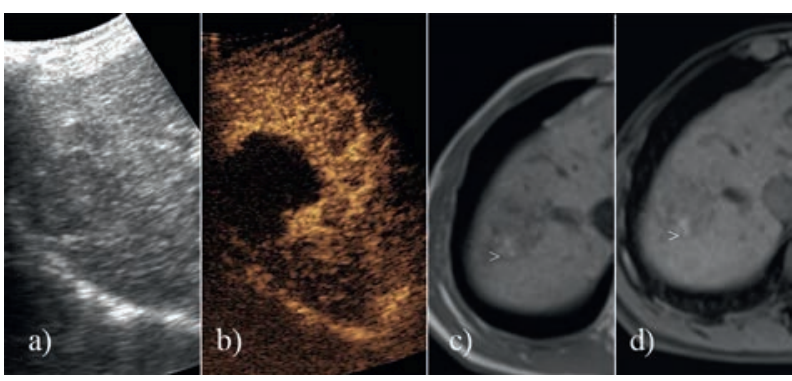

Fig 6. Small residual tumor after TACE in an $4.7 \mathrm{~cm}$ HCC: a) US aspect; b) CEUS revealed a $0.9 \mathrm{~mm}$ enhanced lesion within the necrotic tumor $(>)$; c) T1-weighted images before contrast administration shows a small area of coagulative necrosis of high-signal intensity $(>)$ within the nodule; d) in arterial phase there are no areas of early enhancement within the nodule. The area of coagulative necrosis $(>)$ is unchanged.

Other advantages may arise in cases with impaired enhancement detection at CT (due mainly to lipiodol artefacts) and MRI (TACE related enhancing signal at unenhanced T1) [12].

The combination CT-CEUS could achieve better results in doubtful cases. Last but not least, being non radiating CEUS may decrease the number of CT exams after TACE.

The results of numerous studies have highlighted also the limitations of CEUS. First of all, CEUS is an operator dependent imaging method. Compared to CECT/CEMRI greater intra- and inter- reader variability was found with CEUS [24]. Second, ultrasound detection of liver lesions can be challenging in patients with lesions covered by the lung or diaphragm and in patients with obesity, meteorism, or cirrhosis. Patients with HCC located deep into the liver are considered not suitable for CEUS assessment. Detection of lesions enhancement was found to be impaired at a distance of 8 to $10 \mathrm{~cm}$ from the skin when using Levovist, a $1^{\text {st }}$ generation echo-enhancer [27]. The use of second-generation eco-enhancers like SonoVue may overcome some of these limitations

Tumor multiplicity is another limitation for CEUS mainly to the fact that each lesion has to be studied separately in order to detect changes in its enhancement. However, in one study on a series of fifty-seven patients, CEUS proved efficient for the evaluation of $61.9 \%$ of patients with 2-3 lesions [12]. In our experience three lesions can also be evaluated if at least two of the lesions are located close to each other (fig 7). The additional lesions can be evaluated with another injection of SonoVue, fact that increases the cost. With the new US systems and CEUS software which requires lower amount of contrast agents (up to only $1 \mathrm{ml}$ ) this issue is of less importance.

Hypovascular tumors. Lesions with iso-or hypoenhancing before TACE may be more difficult to be evaluated with CEUS [12]. This problem may be overcome by

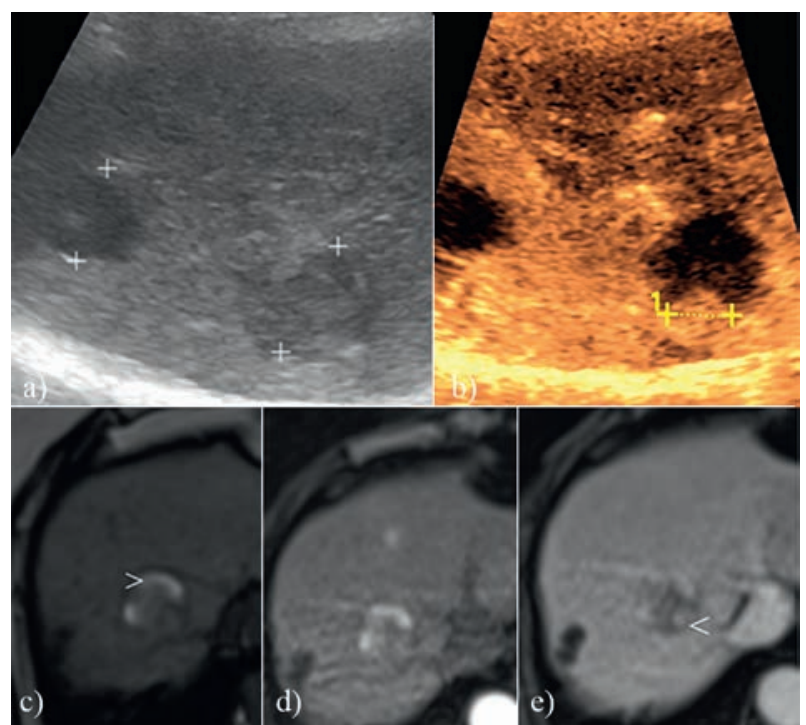

Fig 7. Two HCC lesions after TACE: a) US aspect; b) CEUS was able to assess the vascularity of both lesions with the same contrast agent injection. The lesion in segment VII was necrotic, the other in segment VIII displayed a $1.3 \mathrm{~cm}$ intranodular enhanced area (+); c) T1-weighted out-of-phase image before contrast administration shows a partially necrotic nodule. The areas of coagulative necrosis are high-signal intensity $(>)$; d) In the arterial phase there are no areas of enhancement within the nodul;. e) on the portovenous image there is a small enhancing nodule within the nodule $(>)$. The contrast enhacement's behaviour of the small nodule is not highly suggestive for an HCC. However, based on the CEUS image the nodule was treated by RFA.

the use of dedicated software (time intensity curves TIC), which could quantify lesion enhancement better than visual assessment [12]. This limitation is also present for CT and MRI, in such cases size based criteria like RECIST would be more appropriate.

Other factors. Increased baseline ecogenicity at unenhanced US both in the lesion and in the surrounding liver (steatosis) may impair CEUS evaluation [12]. An increased echogenicity is also present some days after TACE due to post necrotic gas formation or gas bubbles trapped in the embolic material [16]. Post TACE hyperechogenicity will disappear 1 week after TACE.

All these limitations may lead to non-diagnostic pre and post TACE CEUS studies in up to $29.3 \%$ of all HCCs, $14.8 \%$ in single lesions and as high as $41.7 \%$ in more than 2 lesions [12].

\section{New CEUS techniques to evaluate the efficacy of TACE}

Several new CEUS techniques have been developed in order to overcome the current limitations. The experi- 
ence with these new techniques is limited and there is not enough evidence to support their use in daily practice. However they all stand as promising new imaging techniques in HCC management.

Dynamic CEUS with quantification. Uller $\mathrm{W}$ et al evaluated the microcirculation in HCC in vivo by dynamic CEUS after intravenous and intraarterial application of contrast agent during DEB-TACE [28]. The grade of hypervascularization was evaluated before and after Beads application. The percentage of devascularization after Beads application was calculated and quantitative devascularization was carried out using analysis TIC. The results were compared to postinterventional angiography and postprocedural computed tomography. The authors concluded that quantification of the reduction of microvascularization using TIC analysis might be a valuable periinterventional tool during DEB-TACE [28].

Three-dimensional contrast ultrasonography (3DCEUS). In one recent study 2D and 3D CEUS 1 month (30 2 days) after treatment were compared using MRI and $\mathrm{CT}$ as standard of reference. No statistically significant difference between 2D and 3D CEUS in depicting either complete response or residual tumor was found [29]. It should be noted that these are preliminary results and in order to show the theoretical superiority of $3 \mathrm{D}$ CEUS new data is mandatory needed.

Fusion techniques (CEUS-CT, CEUS-MRI) for postinterventional follow-up after TACE in HCC. Ross CJ et al showed that image fusion with volume navigation (VNav) of CEUS with CT or MRI allows an accurate localization of residual tumoral foci in patients with HCC. This exact mapping allows an easier control and evaluation after TACE. Moreover the microcirculation and the residual tumor perfusion are better evaluated at an earlier point of time than usual modalities of therapy control like non-enhanced CT. This approach might lead to a more differentiated monitoring of therapy [30].

\section{Conclusions}

With all its limitations, CEUS is a valuable tool in evaluation the results of TACE. Of all available imaging modalities, CEUS performs better in the early and very early assessment of TACE especially after lipiodol TACE. CEUS performed 5-7 days after TACE could decide whether there is a need for additional treatment. In the standard evaluation after TACE (1-2 months) CEUS seems to perform also better then CECT and at least similar to MRI.

Although the currently available literature does not favor the use of CEUS as a gold standard for the assessment of tumor response after TACE, the excellent results recommend CEUS as an important non-irradiating alternative after TACE. The development of new contrast agents, as well as new software for analyzing sonographic images will evolve. This will create new prospects for characterizing liver lesions and evaluating treatment response.

\section{Conflict of interest: none}

\section{References}

1. Kloeckner R, Otto G, Biesterfeld S, Oberholzer K, Dueber C, Pitton MB. MDCT versus MRI assessment of tumor response after transarterial chemoembolization for the treatment of hepatocellular carcinoma Cardiovasc Intervent Radiol 2010; 33: 532-540.

2. Llovet JM, Burroughs A, Bruix J. Hepatocellular carcinoma. Lancet 2003; 362: 1907-1917.

3. Llovet JM, Bruix J. Systematic review of randomized trials for unresectable hepatocellular carcinoma: Chemoembolization improves survival. Hepatology 2003; 37: 429-442.

4. Takayama T, Makuuchi M, Hirohashi S, et al. Malignant transformation of adenomatous transformation of adenomatous hyperplasia to hepatocellular carcinoma. Lancet 1990; 336: 1150-1153.

5. Minami Y, Kudo M, Kawasaki T, et al. Transcatheter arterial chemoembolization of hepatocellular carcinoma: usefulness of coded phase-inversion harmonic sonography. AJR Am J Roentgenol 2003; 180: 703-708.

6. Bargellini I, Bozzi E, Campani D, et al. Modified RECIST to assess tumor response after transarterial chemoembolization of hepatocellular carcinoma: CT-pathologic correlation in 178 liver explants. Eur J Radiol 2013; 82: e212-e218.

7. Bruix J, Sherman M; American Association for the Study of Liver Diseases. Management of hepatocellular carcinoma: an update. Hepatology 2011; 53: 1020-1022.

8. Lencioni R, Cioni D, Crocetti L, et al. Early- stage hepatocellular carcinoma in patients with cirrhosis: long-term results of percutaneous image-guided radiofrequency ablation. Radiology 2005; 234: 961-967.

9. Nicolau C, Vilana R, Bianchi L, Brú C. Early-stage hepatocellular carcinoma: the high accuracy of real-time contrastenhanced ultrasonography in the assessment of response to percutaneous treatment. Eur Radiol 2007; 17 Suppl 6: F80-F88.

10. Solbiati L, Ierace T, Tonolini M, Cova L. Guidance and monitoring of radiofrequency liver tumor ablation with contrast-enhanced ultrasound. Eur J Radiol 2004; 51 Suppl: S19-S23.

11. Salvaggio G, Campisi A, Lo Greco V, Cannella I, Meloni MF, Caruso G. Evaluation of posttreatment response of hepatocellular carcinoma: comparison of ultrasonography with second-generation ultrasound contrast agent and multidetector CT. Abdom Imaging 2010; 35: 447-453.

12. Moschouris H, Malagari K, Papadaki MG, et al. Short-term evaluation of liver tumors after transarterial chemoemboli- 
zation: limitations and feasibility of contrast-enhanced ultrasonography. Abdom Imaging 2011; 36: 718-728.

13. Minami Y, Kudo M. Imaging modalities for assessment of treatment response to nonsurgical hepatocellular carcinoma therapy: contrast-enhanced US, CT, and MRI. Liver Cancer 2015; 4: 106-114.

14. Kono Y, Lucidarme O, Choi SH, et al. Contrast-enhanced ultrasound as a predictor of treatment efficacy within 2 weeks after transarterial chemoembolization of hepatocellular carcinoma. J Vasc Interv Radiol 2007; 18: 57-65.

15. Xia Y, Kudo M, Minami Y, et al. Response evaluation of transcatheter arterial chemoembolization in hepatocellular carcinomas: the usefulness of sonazoid-enhanced harmonic sonography. Oncology 2008; 75 Suppl 1: 99-105.

16. Moschouris H, Malagari K, Papadaki MG, Kornezos I, Matsaidonis D. Contrast-enhanced ultrasonography of hepatocellular carcinoma after chemoembolisation using drug-eluting beads: a pilot study focused on sustained tumor necrosis. Cardiovasc Intervent Radiol 2010; 33: 10221027.

17. Schacherer D, Girlich C, Zorger N, et al. Sono-hepaticarteriography (Sono-HA) in the assessment of hepatocellular carcinoma in patients undergoing transcatheter arterial chemoembolization (TACE). Ultraschall Med 2010; 31: 270-275.

18. Wiggermann P, Wohlgemuth WA, Heibl M, et al. Dynamic evaluation and quantification of microvascularization during degradable starch microspheres transarterial chemoembolisation (DSM-TACE) of HCC lesions using contrast enhanced ultrasound (CEUS): a feasibility study. Clin Hemorheol Microcirc 2013; 53: 337-348.

19. Morimoto M, Shirato K, Sugimori K, et al. Contrast-enhanced harmonic gray-scale sonographic-histologic correlation of the therapeutic effects of transcatheter arterial chemoembolization in patients with hepatocellular carcinoma. AJR Am J Roentgenol 2003; 181: 65-69.

20. Liu M, Lin MX, Xu ZF et al. Comparison of contrast-enhanced ultrasound and contrast-enhanced computed tomography in evaluating the treatment response to transcatheter arterial chemoembolization of hepatocellular carcinoma using modified RECIST. Eur Rad. 2015; 25: 2502-2511.

21. Cho YZ, Park SY, Choi EH, et al. The usefulness of contrast-enhanced ultrasonography in the early detection of hepatocellular carcinoma viability after transarterial chemoembolization: pilot study. Clin Mol Hepatol 2015; 21: 165-174.
22. Zhao M, Wang JP, Li W, et al. Comparison of safety and efficacy for transcatheter arterial chemoembolization alone and plus radiofrequency ablation in the treatment of single branch portal vein tumor thrombus of hepatocellular carcinoma and their prognosis factors. Zhonghua Yi Xue Za Zhi 2011; 91: 1167-1172.

23. Moschouris H, Malagari K, Kornezos I, Papadaki MG, Gkoutzios P, Matsaidonis D. Unenhanced and contrastenhanced ultrasonography during hepatic transarterial embolization and chemoembolization with drug-eluting beads. Cardiovasc Intervent Radiol 2010; 33: 1215-1222.

24. Shaw CM, Eisenbrey JR, Lyshchik A, et al. Contrastenhanced ultrasound evaluation of residual blood flow to hepatocellular carcinoma after treatment with transarterial chemoembolization using drug-eluting beads: a prospective study. J Ultrasound Med 2015; 34: 859-867.

25. Yan FH, Zhou KR, Cheng JM, et al. Role and limitation of FMPSPGR dynamic contrast scanning in the follow-up of patients with hepatocellular carcinoma treated by TACE. World J Gastroenterol 2002; 8: 658-662.

26. Moschouris H, Malagari K, Papadaki MG, et al. mRECIST criteria and contrast-enhanced US for the assessment of the response of hepatocellular carcinoma to transarterial chemoembolization. Diagn Interv Radiol 2014; 20: 136-142.

27. Youk JH, Lee JM, Kim CS. Therapeutic response evaluation of malignant hepatic masses treated by interventional procedures with contrast-enhanced agent detection imaging. J Ultrasound Med 2003; 22: 911-920.

28. Uller W, Wiggermann P, Gössmann H, et al. Evaluation of the microcirculation of hepatocellular carcinomas using contrast-enhanced ultrasound with intraarterial and intravenous contrast application during transarterial chemoembolization with drug-eluting beads (DEB-TACE): preliminary data. Clin Hemorheol Microcirc 2011; 49: 55-66.

29. Bartolotta TV, Taibbi A, Matranga D, Midiri M, Lagalla R. $3 \mathrm{D}$ versus $2 \mathrm{D}$ contrast-enhanced sonography in the evaluation of therapeutic response of hepatocellular carcinoma after locoregional therapies: preliminary findings. Radiol Med 2015; 120: 695-704.

30. Ross CJ, Rennert J, Schacherer D, et al. Image fusion with volume navigation of contrast enhanced ultrasound (CEUS) with computed tomography (CT) or magnetic resonance imaging (MRI) for post-interventional follow-up after transcatheter arterial chemoembolization (TACE) of hepatocellular carcinomas (HCC): Preliminary results. Clin Hemorheol Microcirc 2010; 46: 101-115. 